\title{
ANALYZING THOUGHT-RELATED ELECTROENCEPHALOGRAPHIC DATA USING NONLINEAR TECHNIQUES
}

\author{
Trent Skidmore \\ Ohio University \\ Athens, Ohio
}

\section{SUMMARY}

A unique method is presented for collecting, studying and interpreting thought-related electroencephalogram (EEG) data. The use of a chaos-based nonlinear analysis technique is shown to be promising in providing insight into relating conscious thought to specific EEG data. A discussion of the practical limitations of this technique is also included.

\section{INTRODUCTION}

The current primary use of the EEG is in clinical applications. However, because the EEG involves conceptually simple instrumentation, is relatively safe, and inherently noninvasive, it is well worth investigating for possible use in other applications. Specifically, if it were possible for a human operator to reliably control some aspect of his/her EEG, a direct humancomputer interface could be developed. Thus, the goal of this research is to determine if an operator can, with their eyes open, consciously control some EEG feature so as to allow a computer to detect one or more bits of information.

In order to achieve this goal, the laboratory set-up shown in figure 1 was created. The host computer is a Macintosh II utilizing National Instruments' LabVIEW software. The interface board is a National Instruments NB-MIO multifunction A/D converter. The EEG amplifier is a Grass Model 8-16C EEG Instrument. The electrodes are $9 \mathrm{~mm}$ silver-silver chloride contacts with collodion adhesive and EEG gel.

The EEG data to be analyzed were obtained during what is called a "Think Math" test. During this test, the subject was instructed to think of a simple math problem (such as $9 \times 8$ $=72$ ) whenever the light-emitting diode (LED) was turned on. The periods when the light is flashing are called Thought-times. During the off-times of the LED (Gap-times), the subject was instructed not to think about mathematics or multiplication. This on/off "Think Math" process was repeated for several minutes with the LED flashing periodically two out of every eight seconds. The data analyzed in this paper were recorded over what is referred to as the "F3 region with respect to $\mathrm{Cz}$ " in the 10-20 International System of Electrode Placement (figure 2). Data were sampled at 128 samples per second $(T=7.8125 \mathrm{~ms})$, well above the Nyquist minimum for data whose highest significant frequency component appears around 15 $\mathrm{Hz}$. 


\section{CHAOS ANALYSIS OF EEG DATA}

The application of chaos theory to biological systems is not a new concept, nor is the study of the chaotic behavior of the EEG. However, the idea of applying chaos theory to determine if the EEG can be used as a thought/non-thought discriminator appears to be new. A fundamental technique in chaos theory is the reinterpretation of nonlinear time signals as multidimensional phase-space geometric objects. The goal is to determine if there exists a "strange attractor" in the phase-space diagram of the multidimensional data. A twodimensional phase-space diagram is generated by plotting the original data sequence, $x(n T)$, against a time-shifted version of the same data, $x(n T+\tau)$. Reference 1 suggests that a time shift $(\tau)$ be used which is equal to one-quarter of the period of the most significant frequency component in the EEG signal. The reasoning for this value is based on the "mutual information content" of a signal. For the analysis in this paper, a time shift of 23.475 milliseconds was chosen. This corresponds to a dominant frequency of $10.6667 \mathrm{~Hz}$. This is intuitively pleasing since the $10.6667 \mathrm{~Hz}$ component is in the middle of the alpha region, considered by some EEG researchers as the most important EEG frequency region.

\section{QUANTIFYING THE STRANGE ATTRACTOR}

Figure 3 shows a phase-space diagram for $x(n T+\tau)$ versus $x(n T)$ for 4 seconds of a 32 second "Think Math" test. In order to quantify the chaotic strange attractor generated during this test, the Standard Deviation/Moment of Inertia (SDMI) transformation was developed. This transformation consists of first finding the point-by-point moment of inertia about the origin of the phase-space "cloud." This is denoted by

$$
I_{x}(n T)=x^{2}\left(n^{\prime} T\right)+x^{2}(n T+\tau) .
$$

The purpose of this transformation is to convert the two-dimensional phase-space data into a positive scalar whose weight is a function of the original signal and the time-shifted version. After this transformation, a moving-average, standard deviation calculation is performed. This is computed as follows:

$$
\begin{aligned}
& \sigma(k)=(1 / m) \sum_{n=1+m k}^{(k+1) m}\left(I_{x}(n T)-\mu_{k}\right)^{2} \quad \text { where: } \\
& \mu_{k}=\sum_{n=1+m k}^{(k+1) m}(1 / m) I_{x}(n T) ; \quad k=0,1,2, \ldots,(N / m)-1
\end{aligned}
$$

where $\mathrm{N}$ is the total length of the data sequence and $\mathrm{m}$ is the standard deviation window length (64 points).

This calculation computes a windowed standard deviation of the moment of inertia of the phase-space "cloud" twice per second (every 64 points). Figure 4 shows the results of this 
analysis by plotting $\sigma(\mathrm{k})$ and the thought-times versus time. Note that this entire run contains four two-second thought times and four six-second gap (non-thought) times.

\section{CONCLUSIONS}

For this subject, it is clear that the SDMI analysis produces a significant peak in the standard deviation plot during the times of transition between thinking and not thinking. In attempting to generalize the results shown here, the entire test was repeated on several other subjects. These subsequent tests, which utilized either visual thought cueing or audio cueing, yielded results very similar to that presented here. Further investigation of the raw EEG did indicate the possibility that unwanted artifact (either eye or muscle movement) contributed to the peaks generated by the SDMI analysis. Thus, some caution is given in interpreting the SDMI peaks as purely thought-related activity. While this research is still in its infancy, it is apparent from these results that this technique may be useful for understanding thoughtrelated EEG data.

\section{REFERENCES}

1. Mayer-Kress, G., and Layne, S.: Dimensionality of the Human Electroencephalogram. Proceedings of the New York Academy of Sciences Conference on Perspectives in Biological Dynamics and Theoretical Medicine, Bethesda, MD, April 9-11, 1986. 


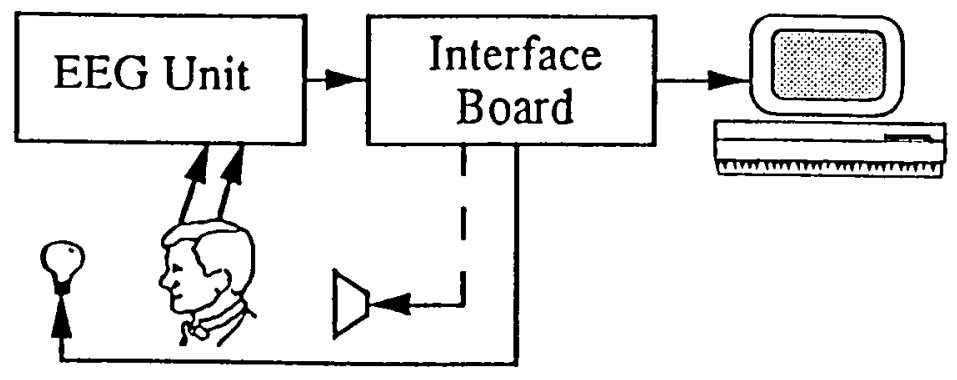

Figure 1. EEG Workstation using a Macintosh II and a Grass Model 8-16C EEG Unit.

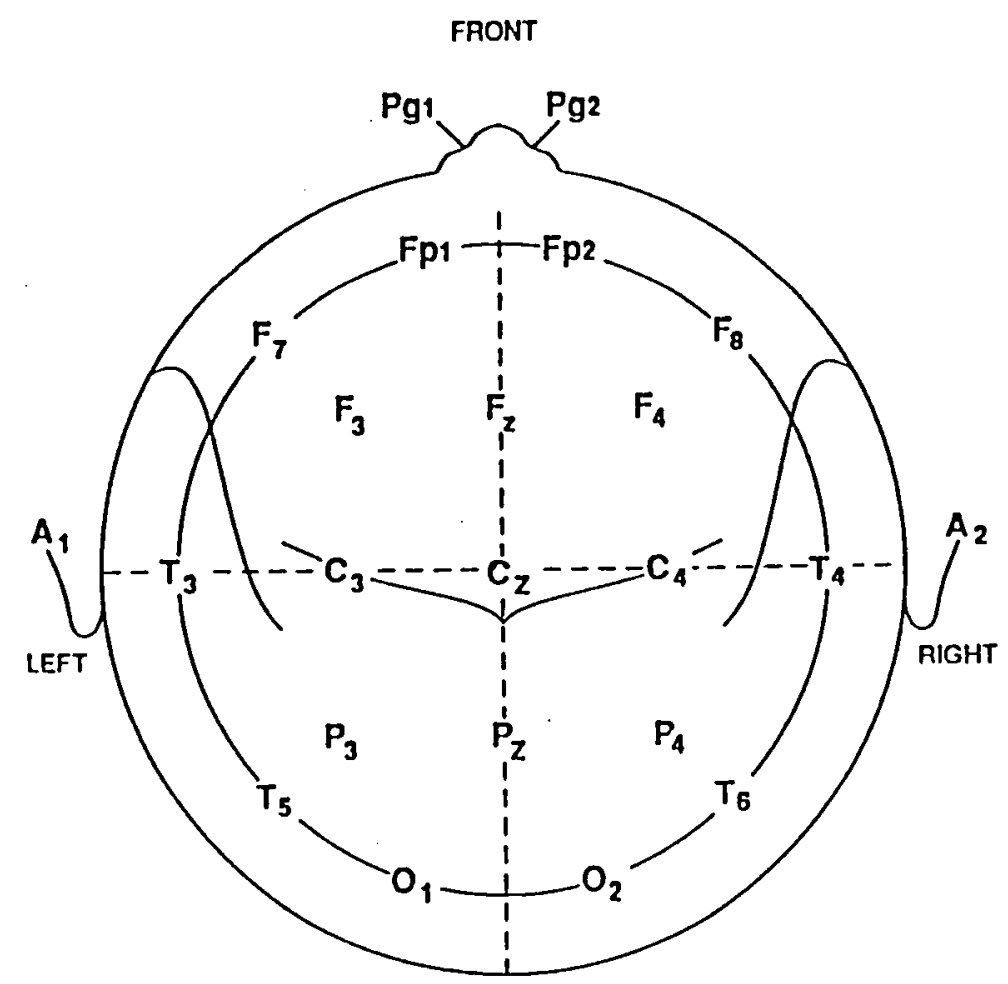

Figure 2. The 10-20 International System of Electrode Placement. 


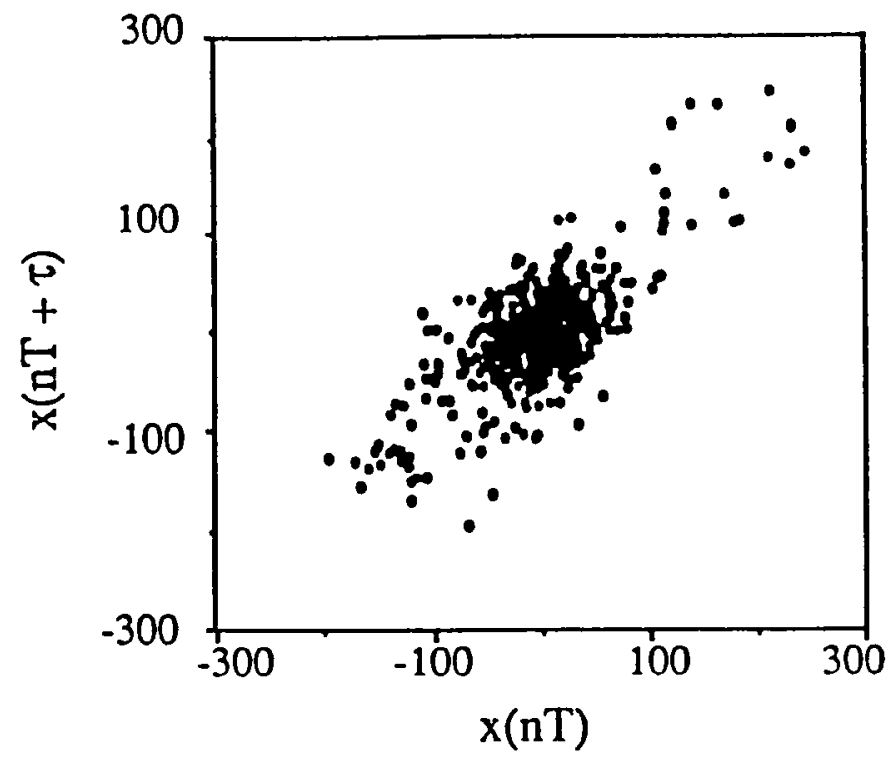

Figure 3. Chaotic Phase-Space "Cloud" showing $x(n T+\tau)$ versus $x(n T)$ for four seconds of data.

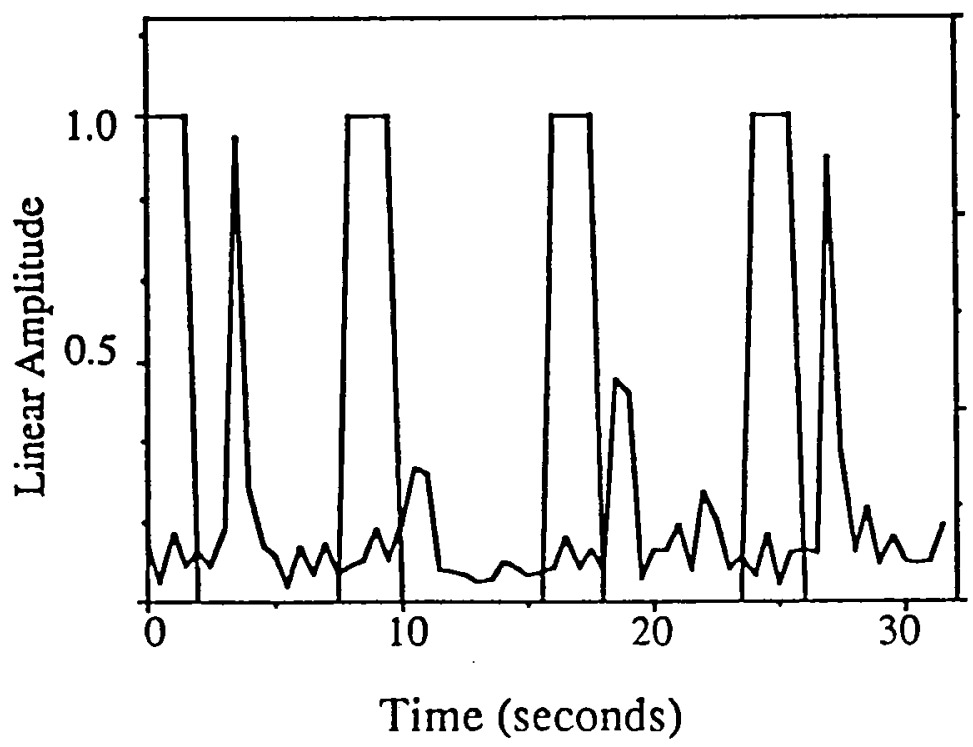

Figure 4. Thought-Times and the SDMI EEG data for a 32 second "Think Math" run. 